\title{
Interleukin 1 receptor antagonist gene variable number of tandem repeats polymorphism and cutaneous melanoma
}

\author{
SABINA CAUCI $^{1}$, CINZIA BULIGAN $^{1,2}$, FRANCESCO ROCCHI ${ }^{1}$, \\ ILARIA SALVADOR $^{1}$, LUIGI XODO ${ }^{1}$ and GIUSEPPE STINCO ${ }^{1,2}$ \\ ${ }^{1}$ Department of Medicine, University of Udine; ${ }^{2}$ Department of Dermatology, \\ University-Hospital of Udine, I-33100 Udine, Italy
}

Received April 24, 2019; Accepted July 11, 2019

DOI: 10.3892/ol.2019.10923

\begin{abstract}
Immunity and cytokines serve crucial roles in cutaneous melanoma. The present study investigated whether a variable number tandem repeat (VNTR) polymorphism of interleukin-1 receptor antagonist (IL-1RA) gene $(I L-1 R N)$ located in intron 2 (rs2234663) is associated with cutaneous melanoma. A total of 515 subjects were studied, 133 of which were cutaneous melanoma cases (72 stage I+II non-metastatic melanoma cases and 61 stage III+IV metastatic melanoma cases), and 382 subjects were matching healthy controls from the Friuli-Venezia-Giulia Region located in Northeast Italy, an area with a high melanoma incidence. The $I L-I R N$-VNTR polymorphism was determined by DNA fragment length analysis following PCR amplification. According to the number of 86 -bp repeats, five different $I L-1 R N$ alleles were identified: Allele 1 (4-repeats), allele 2 (2-repeats, short allele), allele 3 (5-repeats), allele 4 (3-repeats) and allele 5 (6-repeats). Alleles with three or more 86 -bp repeats, i.e. allele 1, 3, 4 and 5 were collectively denoted as long (L) repeats. The present study revealed that $I L-1 R N-\mathrm{VNTR} 1 / 2$ and $2 / \mathrm{L}$ genotypes were more frequent among patients with cutaneous melanoma (43.6 and $45.1 \%$, respectively) compared with healthy controls [29.6 and 30.6\%, respectively; odds ratio (OR), 1.84; CI, 1.22-2.77; $\mathrm{P}=0.003$; and $\mathrm{OR}, 1.66$; CI, 1.24-2.79; $\mathrm{P}=0.002$, respectively]. Conversely, the $I L-1 R N-\mathrm{VNTR} 1 / 1$ genotype was less frequent among melanoma cases (45.9\%) compared with healthy controls (57.9\%; OR, 0.62; CI, 0.41-0.92; $\mathrm{P}=0.017)$.
\end{abstract}

Correspondence to: Dr Sabina Cauci, Department of Medicine, University of Udine, Piazzale Kolbe 4, I-33100 Udine, Italy

E-mail: sabina.cauci@uniud.it

Abbreviations: IL-1RA, interleukin 1 receptor antagonist; IL-1RN, interleukin 1 receptor antagonist gene; VNTR, variable number of tandem repeats polymorphism; FVG, Friuli Venezia-Giulia; MetM, metastatic melanoma; NMetM, non-metastatic melanoma; BMI, body mass index; TILs, tumor infiltrating lymphocytes

Key words: cutaneous melanoma, skin cancer, polymorphism, interleukin 1 family, interleukin 1 receptor, interleukin 1 receptor antagonist, immunity, innate response
Comparison of metastatic vs. non-metastatic melanoma cases identified no significant differences. The present study first demonstrated that carriage of the $1 / 1 I L-1 R N-V N T R$ genotype was protective, whereas $1 / 2$ and $2 / \mathrm{L}$ was a risk factor for patients with cutaneous melanoma vs. healthy controls. The short allele 2 was associated with higher expression levels of IL-1RA, a potent competitive inhibitor of the proinflammatory cytokines IL-1 $\alpha$ and IL-1 $\beta$. VNTR- $I L-1 R N$ polymorphism may affect susceptibility to melanoma and, thus, it is a potential novel diagnostic biomarker for melanoma. The present study increased the understanding of genetic melanoma susceptibility/carcinogenesis, and may indicate novel strategies in the personalized prevention of cutaneous melanoma.

\section{Introduction}

Factors that affect cutaneous melanoma need to be addressed and molecular genetic studies appear promising for a precision/personalized medicine approach (1-3). A growing body of data indicates that besides exposure to ultraviolet (UV) radiation, especially intermittent sun exposure (4), sunburns $(4,5)$, and fair skin (4-6), other factors including vitamin D (2,3,7), polychlorinated biphenyls $(8)$, host inflammatory responses $(9,10)$, geographical factors $(5,6,11,12)$, the lifestyle (11-13), and genetic background can also play a role $(1-3,14)$. Melanoma has a rate of 22.0 in males and 18.3 in females per 100,000/year (standardized for European population) in Italy (15), and higher incidence rates (32.5/100,000/year in males; 23.7/100,000/year in females) have been recorded in Friuli Venezia Giulia (FVG) (15), a Region located in Northeast Italy at the border with Austria and Slovenia. The incidence of melanoma in Italy shows an increasing trend (15), Northeast Italy has the highest prevalence of melanoma (16), and the FVG Region has the highest rate of hospitalization for melanoma in Italy (17).

The interleukin 1 alpha (IL-1 $\alpha$ ) and IL-1 beta (IL-1 $\beta$ ) cytokines and the specific receptor antagonist (IL-1RA) are components of the interleukin 1 family (18). IL-1 $\alpha$ and IL-1 $\beta$ are involved in several physiological and pathological diseases $(18,19)$, modulating the immune response to pathogenesis and tissue injuries. Moreover, these cytokines have a role in the promotion of cancer-associated inflammation paradoxically both as protective or favoring cancer/metastasis onset $(19,20)$. IL-1RA neutralizes IL- $1 \alpha$ and IL-1 $\beta$ action by 
binding to the IL-1 receptors (IL-1R type I, IL-1R1 and type II, IL-1R2) without causing any signaling transduction (18). This prevents the IL-1 $\alpha$ and IL-1 $\beta$ induced cascade of kinases that activates NF-kB and cyclooxygenase-2 (COX-2) following IL-1 (either $\alpha$ or $\beta$ ) binding to IL-1R1 (18). The IL-1 family of cytokines and receptors is involved in a broad spectrum of immunological and inflammatory responses including activation of lymphocytes (18-22). Specifically, IL-1 cytokines promote increased levels of the main chemokine IL-8 (CXCL8) and consequently activate recruitment of neutrophils $(23,24)$. An in vitro human cellular study showed that IL-1RA inhibits CXCL8 release (24). Interestingly, a recent research in myeloid cells found that inflammasomes/IL-1 pathways induce the expression of the programmed death-ligand 1 (PD-L1) on tumor cells, which is an immune checkpoint molecule used as target of melanoma therapy $(25,26)$. Furthermore, blocking IL-1 receptor with IL-1RA or anti-IL-1R1 antibody inhibits tumor growth and metastasis accompanied by decreased accumulation of myeloid cells and expression of the PD-L1 molecule (25).

Currently, inflammation and specific IL-1 targeting as treatment of cancer is an active area of experimental and clinical research $(18,20,27)$. IL-1 mediated inflammation is proposed to contribute to the development and progression of some cancers including melanoma (28). IL-1 appear to act at different levels in tumor initiation and progression, including driving chronic non-resolving inflammation, tumor angiogenesis, activation of the IL-17 pathway, induction of myeloid-derived suppressor cells and macrophage recruitment, invasion and metastasis (20). The effects of IL-1 cytokines are pleiotropic so that any shift of the biological balance between agonistic and antagonistic signals has the potential to cause a disease (19). Notably, the role of IL-1RA in cancer has been studied in different types of tumors showing effects on survival and progression (18-22).

Increasing evidence showed that genetic polymorphisms of IL-1 family members can affect susceptibility to disease. Human IL-1RA gene $(I L-I R N)$ is located in chromosome 2, specifically 2q13-2q21 $(29,30)$. Current studies and meta-analyses evaluated the role of a variable number of tandem (VNTR) 86-bp repeats located in intron 2 of the $I L-I R N$ gene in relation to various diseases and cancers, particularly gastric cancer (31-33). Specifically, the $I L-1 R N$ allele $2(I L-1 R N * 2$, constituted of two 86-bp repeats also denoted as short allele) has been associated with increased cancer risk in heterozygous subjects (33). Nonetheless, $I L-1 R N$ VNTR polymorphism roles in cancer still require further study as inconsistent results have been obtained in cancers of different tissue origins (33).

So far, only one German study assessed the role of $I L-1 R N$ VNTR polymorphism in 97 melanoma patients and 343 controls (34). No significant findings were obtained, the heterozygous $1 / 2$ genotype was found in $28.8 \%$ of advanced melanoma patients vs. $39.6 \%$ of healthy controls; $\mathrm{P}=0.06$ (34).

Pathways underlying the relationship of $I L-I R N$ VNTR and melanoma currently poses an interesting new challenge in melanoma research $(1,34)$ also by considering the potential roles of IL-1RA in modulating PD-1/PD-L1 (25).

Progress in the understanding of melanoma risk factors, genomics, and molecular pathogenesis may drive advances in precision medicine applied to melanoma $(1-3,35,36)$.
In light of these observations, we explored $I L-1 R N$ VNTR polymorphism and its association with cutaneous malignant melanomas, specifically those with metastatic melanoma (MetM) vs. non-metastatic melanoma (NMetM) and vs. healthy controls.

\section{Materials and methods}

Population. Enrolment and clinical visits of all study participants were performed at the Udine University-Hospital Dermatology Clinic. Diagnostic procedures were carried out according to routine protocols. The Udine Institutional Ethical Committee approved the study protocol, which was conducted according to the Declaration of Helsinki. All participants were alive during enrolment in the study and signed a written informed consent.

Using a case-control design, the study consecutively enrolled 133 (age range of 31-87 years) unrelated patients (hospitalized or outpatients) of both sexes with documented cutaneous melanoma diagnosis and 382 (age range of 31-87 years) asymptomatic healthy controls of both sexes, which were matched for age and ancestry with melanoma cases. Inclusion criteria for both melanoma cases and healthy controls were as follows: Caucasian resident in FVG Region, at least two Italian grandparents born in FVG Region (or Austro-Hungarian territory before World War I) as described $(2,3)$. Due to the demonstrated association of $I L-I R N$ VNTR with high-grade athleticism, athletes were excluded both from melanoma cases and healthy controls as described (37). Further, exclusion criteria for healthy controls included the following: any kind of lifelong malignant or benign tumor, first-grade relatives with a history of melanoma, and major chronic diseases, such as autoimmune diseases including type 1 diabetes. Among healthy controls 268 subjects were previously studied (37).

Melanoma was diagnosed using immunohistological findings obtained after surgical excision of nevi with clinical and dermoscopic characteristics suggesting the presence of malignancy. Classification of melanoma stages was performed by clinical/histological/radiological findings, as described $(38,39)$, which was our routine in the period of the study whose patient enrollment was completed on December 2017. Inclusion criteria for case-patients comprised only cutaneous not mucosal melanomas. For patients with multiple melanomas, the major melanoma characteristics were accounted for in study analyses according to the histological assessment of major primary tumor $(\mathrm{T})$ grading.

Each participant answered a questionnaire, which was used to collect data on demographic characteristics, medical and family history of melanoma, smoking habits, and history of sunburns as described (2). Phototype was assessed by Fitzpatrick criteria (39). BMI was determined by weight $(\mathrm{kg})$ divided by squared height $\left(\mathrm{m}^{2}\right)$; BMI $>30 \mathrm{~kg} / \mathrm{m}^{2}$ was considered an indicator of obesity.

Genetic analysis of the VNTR IL-IRN polymorphism. VNTR IL-IRN polymorphism was determined, as previously described (40) after extraction of genomic DNA from ethylenediaminetetraacetic-acid-treated venous blood samples (41). The $I L-I R N$ intron 2 VNTR polymorphism (rs2234663; also indicated as rs380092) was analyzed using 5'-CTCAGCAAC 
ACTCCTAT-3' and 5'-TCCTGGTCTGCAGGTAA-3' as primers $(37,40)$. The PCR products of 412-bp (allele 1 corresponding to 4 repeats of the 86-bp region), 240-bp (allele 2, 2 repeats also defined as short allele), 498-bp (allele 3, 5 repeats), 326-bp (allele 4, 3 repeats), 584-bp (allele 5, 6 repeats) were analyzed by electrophoresis on $10 \%$ acrylamide gel stained with ethidium bromide (40). Alleles with three or more 86-bp repeats were denoted as long (L) alleles (37).

Statistical analysis. Continuous variables were expressed as a mean \pm standard deviation, and Mann-Whitney $U$ test was performed for comparison. Odds ratios (ORs) and 95\% confidence intervals (CIs) were calculated for categorical variables, and $\mathrm{P}$ values for two-sided Pearson's chi-squared or Fisher's exact test were reported as was appropriate.

$\mathrm{P}<0.05$ was considered to indicate a statistically significant difference and $\mathrm{P} \leq 0.10$ indicates a tendency to be significant. Statistical software SPSS for Windows (SPSS Inc., Chicago, IL, USA) was used.

\section{Results}

Study subjects. All 515 (133 cutaneous melanoma patients and 382 healthy controls) study subjects were Italian Caucasian residents in Northeast Italy. Melanoma cases and healthy controls did not differ for age.

Comparison of VNTR IL-1RN genotypes in cutaneous melanoma patients and healthy controls (as shown in Table I). In all 515 study subjects (data shown in Table SI), the most frequent $I L-I R N$ allele was allele $1\left(I L-I R N^{*} 1\right.$, frequency was $72.9 \%)$, followed by allele $2(I L-1 R N * 2$, frequency was $24.5 \%)$ and by allele $3\left(I L-1 R N^{*} 3\right.$, frequency was $\left.2.5 \%\right)$. The rare heterozygous genotype $1 / 4$ was found in one MetM patient, and 1/5 genotype was found in one NMetM patient, finally, the rare homozygous $3 / 3$ was found in one healthy control.

Homozygous $1 / 1$ genotype was less frequent in melanomas than in healthy control subjects $(\mathrm{OR}=0.62, \mathrm{P}=0.017)$. Conversely, heterozygous $1 / 2$ genotype was almost twice more frequent in melanomas than in healthy patients $(\mathrm{OR}=1.84$, $\mathrm{P}=0.003)$. No differences were observed for $1 / 3,2 / 2$, and $2 / 3$ genotypes. The group of all heterozygous genotypes containing the short allele 2 and one of the long alleles 1, 3 , 4, 5 (designed in the literature as $2 / \mathrm{L}$ genotype) $(33,37)$ had $\mathrm{OR}=1.66, \mathrm{P}=0.002$ for melanomas compared with healthy controls. The group including all genotypes containing the short allele 2 (heterozygous $2 / \mathrm{L}$ plus homozygous $2 / 2$ genotypes) was more frequent in melanoma patients than in healthy controls $(\mathrm{OR}=1.53, \mathrm{P}=0.036)$.

As shown in Table I, frequency of allele 1, 2, and 3 did not significantly differ between melanoma and healthy groups, although carriers of allele 1 were slightly less frequent among melanoma patients $(70.3 \%)$ than healthy controls (74.3\%), $\mathrm{OR}=0.82, \mathrm{P}=0.199$, whereas carriers of the allele 2 were slightly more frequent among melanoma patients (26.3\%) than healthy controls $(23.2 \%), \mathrm{OR}=1.18, \mathrm{P}=0.300$.

Comparisons of IL-1RN VNTR genotypes in MetM and NMetM cutaneous melanoma patients and healthy controls (as shown in Table II). The VNTR IL-IRN genotypes were not associated with the metastatic grade of cutaneous melanoma as illustrated in Table II by comparison of MetM with NMetM patients.

By comparison of 61 MetM patients with healthy controls, $1 / 1$ genotype was protective $(\mathrm{OR}=0.58, \mathrm{P}=0.047)$ and $1 / 2$ was at risk $(\mathrm{OR}=1.77, \mathrm{P}=0.042)$, the group of $2 / \mathrm{L}$ genotypes showed a tendency at risk $(\mathrm{OR}=1.68, \mathrm{P}=0.063)$.

By comparison of 72 NMetM patients with healthy controls, the $1 / 2$ and $2 / \mathrm{L}$ genotypes were at risk $(\mathrm{OR}=1.90$, $\mathrm{P}=0.013$, and $\mathrm{OR}=2.03, \mathrm{P}=0.006$, respectively).

The frequency of allele 1,2, and 3 did not differ between groups as shown in Table II.

Primary demographic and clinical characteristics of melanoma patients and comparison between VNTR IL-IRN 2/L and other remaining genotypes (as shown in Table III). Main demographic and clinical characteristics of the 133 melanoma patients are described in Table III. We examined in detail the differences between heterozygous carriers of the short allele 2 $(2 / \mathrm{L}$ group, $\mathrm{n}=60)$ and the other remaining genotypes (non- $2 / \mathrm{L}$ group, $\mathrm{n}=73$ ) in melanoma patients. No differences were noted between groups, however, the frequency of patients with a number of body nevi $>50$ tended to be higher in $2 / \mathrm{L}$ than non- $2 / \mathrm{L}$ carriers $(58.3 \%$ vs. $42.5 \%, \mathrm{OR}=1.90, \mathrm{P}=0.069)$.

In a further analysis (data are not shown) by comparison of $2 / \mathrm{L}$ carriers with $\mathrm{L} / \mathrm{L}$ carriers $(\mathrm{n}=68)$ among melanoma patients, still non significant differences were noted, however, frequency of patients with a number of body nevi $>50$ tended to be higher in $2 / \mathrm{L}$ than $\mathrm{L} / \mathrm{L}$ carriers $(58.3 \%$ vs. $42.6 \%, \mathrm{OR}=1.88$, $\mathrm{CI}=0.93-3.80, \mathrm{P}=0.077$ ).

Complete questionnaire data including body nevi number were available for 114 healthy control subjects; no significant differences were noted for a nevi number $>50$ by comparing $2 / \mathrm{L}$ vs. all other remaining genotypes $(12 / 40,30.0 \%$ vs. $25 / 74$, $33.8 \%, \mathrm{OR}=0.84, \mathrm{CI}=0.37-1.93, \mathrm{P}=0.680$ ), and by comparing $2 / \mathrm{L}$ vs. $\mathrm{L} / \mathrm{L}(12 / 40,30.0 \%$ vs. $23 / 66,34.8 \%, \mathrm{OR}=0.80$, $\mathrm{CI}=0.34-1.86, \mathrm{P}=0.607)$.

\section{Discussion}

IL-1RA has been implicated in oncogenesis as mice deficient in IL-1RA develop the disease in response to carcinogens (42). IL-1RA by binding to IL-1R1 strongly modulates the action of IL-1 (IL- $1 \alpha$ and IL-1 $\beta$ ) cytokines, which in turn can induce expression of hundreds of genes, including themselves (via a positive feedback loop) (43).

$I L-1 R N$ VNTR polymorphism. IL-IRN is considered an important 'hub' gene in melanoma research (1). Previous studies found associations of the short $I L-I R N^{*} 2$ allele (two 86-bp repeats) with a variety of epithelial-related chronic inflammatory diseases including psoriasis, scleroderma, alopecia areata, lichen sclerosus, systemic lupus erythematosus, and ulcerative colitis (44).

The number of 86-bp repeats in the VNTR $I L-1 R N$ polymorphism, which is located in intron 2 , does not alter the encoded amino acid sequence of IL-1RA, but may be of functional significance as the repeated sequences contain putative binding sites for transcription factors $(45,46)$. A 
Table I. Genotype frequencies of $I L-1 R N$ VNTR in all 515 study subjects and comparison of 133 patients with melanoma with 382 healthy controls.

\begin{tabular}{lccccc}
\hline $\begin{array}{l}\text { IL- } 1 R N \text { VNTR } \\
\text { genotype/allele }\end{array}$ & $\begin{array}{c}\text { All subjects } \\
(\mathrm{n}=515)(\%)\end{array}$ & $\begin{array}{c}\text { All patients with } \\
\text { melanoma }(\mathrm{n}=133)(\%)\end{array}$ & $\begin{array}{c}\text { Healthy controls } \\
(\mathrm{n}=382)(\%)\end{array}$ & $\begin{array}{c}\text { OR } \\
(95 \% \mathrm{CI})\end{array}$ & $\begin{array}{c}\text { P-value } \\
\text { Melanoma vs. Control }\end{array}$ \\
\hline $1 / 1$ & $282(54.8)$ & $61(45.9)$ & $221(57.9)$ & $0.62(0.41-0.92)$ & 0.017 \\
$1 / 2$ & $171(33.2)$ & $58(43.6)$ & $113(29.6)$ & $1.84(1.22-2.77)$ & 0.003 \\
$1 / 3$ & $18(3.5)$ & $5(3.8)$ & $13(3.4)$ & $1.11(0.39-3.17)$ & 0.789 \\
$2 / 2$ & $35(6.8)$ & $5(3.8)$ & $30(7.9)$ & $0.46(0.17-1.21)$ & 0.106 \\
$2 / 3$ & $6(1.2)$ & $2(1.5)$ & $4(1.0)$ & $1.44(0.26-7.97)$ & 0.651 \\
$2 / \mathrm{L}(1 / 2$ and 2/3) & $177(34.4)$ & $60(45.1)$ & $117(30.6)$ & $1.66(1.24-2.79)$ & 0.002 \\
$1 / 2$ and $2 / 2$ and 2/3 and $2 / 4$ & $212(41.2)$ & $65(48.9)$ & $147(38.5)$ & $1.53(1.03-2.27)$ & 0.036 \\
$1 / 3$ and 2/3 and 3/3 & $25(4.9)$ & $7(5.3)$ & $18(4.7)$ & $1.12(0.46-2.75)$ & 0.799 \\
Allele 1 & $755(73.3)^{\mathrm{c}}$ & $187(70.3)^{\mathrm{c}}$ & $568(74.3)$ & $0.82(0.60-1.11)$ & 0.199 \\
Allele 2 & $247(24.0)$ & $70(26.3)$ & $177(23.2)$ & $1.18(0.86-1.63)$ & 0.300 \\
Allele 3 & $26(2.5)^{\mathrm{d}}$ & $7(2.6)$ & $19(2.5)^{\mathrm{d}}$ & $1.06(0.44-2.55)$ & 0.897 \\
\hline
\end{tabular}

${ }^{\text {a}}$ Heterozygous subjects containing the short allele 2 and one long allele (1, 3, 4 and 5) are denoted as 2/L according the literature (33). ${ }^{b}$ Homozygous genotype $3 / 3$ was present in only one healthy subject. ${ }^{c}$ One metastatic patients had genotype $1 / 4$, and one non-metastatic patient had genotype $1 / 5$. ${ }^{\mathrm{d}}$ One healthy subject had genotype 3/3. IL-IRN, interleukin-1 receptor antagonist gene; OR, odds ratio; VNTR, variable number tandem repeat.

Table II. Genotype frequencies of $I L-1 R N$ VNTR comparisons of 61 patients with MetM and 72 patients with NMetM and 382 healthy controls.

\begin{tabular}{|c|c|c|c|c|c|c|c|c|}
\hline $\begin{array}{l}I L-I R N \text { VNTR } \\
\text { genotype/allele }\end{array}$ & $\begin{array}{c}\text { MetM } \\
(\mathrm{n}=61)(\%)\end{array}$ & $\begin{array}{c}\text { NMetM } \\
(\mathrm{n}=72)(\%)\end{array}$ & $\begin{array}{c}\mathrm{OR}^{\mathrm{a}} \\
(95 \% \mathrm{CI})\end{array}$ & $\mathrm{P}$-value ${ }^{\mathrm{a}}$ & $\begin{array}{c}\mathrm{OR}^{\mathrm{b}} \\
(95 \% \mathrm{CI})\end{array}$ & P-value ${ }^{b}$ & $\begin{array}{c}\mathrm{OR}^{\mathrm{c}} \\
(95 \% \mathrm{CI})\end{array}$ & P-value \\
\hline $1 / 1$ & $27(44.3)$ & $34(47.2)$ & $0.89(0.45-1.76)$ & 0.733 & $0.58(0.34-1.00)$ & 0.047 & $0.65(0.39-1.08)$ & $0.095^{\mathrm{g}}$ \\
\hline $1 / 2$ & $26(42.6)$ & $32(44.4)$ & $0.93(0.47-1.85)$ & 0.833 & $1.77(1.02-3.07)$ & 0.042 & $1.90(1.14-3.18)$ & 0.013 \\
\hline $1 / 3$ & $3(4.9)$ & $2(2.8)$ & $1.81(0.29-11.2)$ & 0.660 & $1.47(0.41-5.31)$ & 0.472 & $0.81(0.18-3.67)$ & 1.000 \\
\hline $2 / 2$ & $4(6.6)$ & $1(1.4)$ & $4.98(0.54-45.8)$ & 0.179 & $0.82(0.28-2.42)$ & 1.000 & $0.16(0.02-1.23)$ & 0.043 \\
\hline $2 / 3$ & $0(-)$ & $2(2.8)$ & $0.97(0.93-1.01)$ & 0.500 & $0.99(0.98-1.00)$ & 1.000 & $2.70(0.48-15.0)$ & 0.243 \\
\hline $2 / \mathrm{L}(1 / 2 \text { and } 2 / 3)^{\mathrm{f}}$ & $26(42.6)$ & $34(47.2)$ & $0.83(0.42-1.65)$ & 0.595 & $1.68(0.97-2.92)$ & $0.063^{\mathrm{g}}$ & $2.03(1.21-3.38)$ & 0.006 \\
\hline $\begin{array}{l}1 / 2 \text { and } 2 / 2 \text { and } \\
2 / 3 \text { and } 2 / 4\end{array}$ & $30(49.2)$ & $35(48.6)$ & $1.02(0.52-2.02)$ & 0.948 & $1.55(0.90-2.66)$ & 0.113 & $1.51(0.91-2.51)$ & 0.108 \\
\hline $1 / 3$ and $2 / 3$ and $3 / 3$ & $3(4.9)$ & $4(5.5)$ & $0.88(0.19-4.09)$ & 1.000 & $1.05(0.30-3.66)$ & 1.000 & $1.19(0.39-3.62)$ & 0.764 \\
\hline Allele 1 & $84^{\mathrm{d}}(68.9)$ & $103^{\mathrm{e}}(71.5)$ & $0.88(0.52-1.49)$ & 0.634 & $0.76(0.50-1.16)$ & 0.201 & $0.87(0.58-1.29)$ & 0.480 \\
\hline Allele 2 & $34(27.9)$ & $36(25.0)$ & $1.16(0.67-2.00)$ & 0.596 & $1.28(0.83-1.97)$ & 0.258 & $1.10(0.73-1.67)$ & 0.634 \\
\hline Allele 3 & $3(2.5)$ & $4(2.8)$ & $0.88(0.19-4.02)$ & 1.000 & $0.99(0.29-3.39)$ & 1.000 & $1.12(0.37-3.34)$ & 0.774 \\
\hline
\end{tabular}

${ }^{\mathrm{a} C}$ Comparison between MetM and NMetM. ${ }^{\mathrm{b}}$ Comparison between MetM and healthy controls. ${ }^{\mathrm{c}}$ Comparison between NMetM and healthy controls. ${ }^{\mathrm{d}}$ One metastatic patients had genotype $1 / 4$. ${ }^{\mathrm{e}}$ One non-metastatic patient had genotype $1 / 5 .{ }^{\mathrm{f}}$ Heterozygous subjects containing the short allele 2 and one long allele are denoted as $2 / \mathrm{L}$ in the literature (33). ${ }^{\mathrm{g}}$ Significant tendencies. $I L-1 R N$, interleukin-1 receptor antagonist gene; MetM, metastatic melanoma; NMetM, non-metastatic melanoma; OR, odds ratio; VNTR, variable number tandem repeat.

general correlation between $I L-1 R N^{*} 2$ allele and the presence of autoinflammatory disease strongly supports a role of VNTR $I L-1 R N$ polymorphism in the control of the inflammatory response (46).

We observed a general distribution of $I L-1 R N$ VNTR genotypes $(1 / 1,54.8 \% ; 1 / 2,33.2 \% ; 1 / 3,3.5 \% ; 2 / 2,6.8 \%$; $2 / 3,1.2 \% ; 1 / 4,0.2 \% ; 1 / 5,0.2 \%$ and $3 / 3,0.2 \%$ ) among the 515 Italian study subjects roughly in agreement with other investigations $(33,34,40)$.
Notably, in our study, nearly twofold higher frequency of $1 / 2$ VNTR $I L-1 R N$ genotype was observed in melanoma patients $(43.6 \%)$ compared with healthy controls $(29.6 \%)$, $\mathrm{OR}=1.84, \mathrm{P}=0.003$. This $\mathrm{OR}$ value became slightly lower (but still statistically significant) by grouping all heterozygous genotypes containing the short 2 allele and one long allele $(2 / \mathrm{L}$ group, $\mathrm{OR}=1.66, \mathrm{P}=0.002)$, and all carriers of allele $2(2 / \mathrm{L}+2 / 2)$ $(\mathrm{OR}=1.53, \mathrm{P}=0.036)$. We observed that the $1 / 2$ genotype was still at risk by comparing separately the subgroups of metastatic 
Table III. Clinical characteristics of 133 patients with melanoma consecutively enrolled, and comparison between the two genetic subgroups of $I L-1 R N$ VNTR 2/L genotypes $(\mathrm{n}=60)$ and all other genotypes $(\mathrm{n}=73)$.

\begin{tabular}{|c|c|c|c|c|c|}
\hline Characteristics & $\begin{array}{l}\text { All patients with } \\
\text { melanoma }(n=133)\end{array}$ & $\begin{array}{l}\text { 2/L genotype } \\
\quad(n=60)\end{array}$ & $\begin{array}{c}\text { Non- } 2 / \mathrm{L} \\
\text { genotypes }(n=73)\end{array}$ & $\begin{array}{c}\text { OR }(\mathrm{CI}) \\
\text { 2/L vs. Non-2/L }\end{array}$ & $\begin{array}{l}\text { P-value, } \\
\text { 2/L vs. Non-2/L }\end{array}$ \\
\hline Age $<50$ years, $\mathrm{n}(\%)$ & $29(21.8)$ & $13(21.7)$ & $16(21.9)$ & $0.98(0.43-2.25)$ & 0.972 \\
\hline $\begin{array}{l}\text { Age at study enrolment, } \\
\text { years, mean } \pm \text { SD }\end{array}$ & $60.8 \pm 12.7$ & $61.1 \pm 12.9$ & $60.7 \pm 12.7$ & - & $0.788^{\mathrm{a}}$ \\
\hline $\begin{array}{l}\text { Age at melanoma diagnosis, } \\
\text { years, mean } \pm \mathrm{SD}\end{array}$ & $54.2 \pm 13.7$ & $54.4 \pm 13.5$ & $54.0 \pm 14.0$ & - & $0.781^{\mathrm{a}}$ \\
\hline $\begin{array}{l}\text { Time from melanoma diagnosis, } \\
\text { years, mean } \pm \mathrm{SD}\end{array}$ & $6.7 \pm 4.1$ & $6.7 \pm 4.4$ & $6.6 \pm 3.9$ & - & $0.754^{\mathrm{a}}$ \\
\hline Females, n $(\%)$ & $58(43.6)$ & $26(43.3)$ & $32(43.8)$ & $0.98(0.49-1.95)$ & 0.954 \\
\hline Males, n (\%) & $75(56.4)$ & $34(56.7)$ & $41(56.2)$ & $1.02(0.51-2.03)$ & 0.954 \\
\hline All grand-parents born in FVG & $96(72.2)$ & $43(71.7)$ & $53(72.6)$ & $0.95(0.45-2.04)$ & 0.905 \\
\hline $\mathrm{BMI}, \mathrm{kg} / \mathrm{m}^{2}$, mean $\pm \mathrm{SD}$ & $25.8 \pm 3.97$ & $26.1 \pm 4.0$ & $25.6 \pm 4.0$ & - & $0.643^{\mathrm{a}}$ \\
\hline $\mathrm{BMI}>30 \mathrm{~kg} / \mathrm{m}^{2}, \mathrm{n}(\%)$ & $22(16.5)$ & $11(18.3)$ & $11(15.1)$ & $1.26(0.51-3.16)$ & 0.614 \\
\hline High school, n (\%) & $60(45.1)$ & $27(45.0)$ & $33(45.2)$ & $0.99(0.50-1.97)$ & 0.981 \\
\hline Graduation, n (\%) & $15(11.3)$ & $5(8.3)$ & $10(13.7)$ & $0.57(0.18-1.78)$ & 0.330 \\
\hline Present smoker, n (\%) & $12(9.0)$ & $4(6.7)$ & $8(11.0)$ & $0.58(0.17-2.03)$ & 0.390 \\
\hline Past smoker, n (\%) & $53(39.8)$ & $25(41.7)$ & $28(38.4)$ & $1.15(0.57-2.30)$ & 0.698 \\
\hline Ever smoker, n (\%) & 65 (48.9) & $29(48.3)$ & $36(49.3)$ & $0.96(0.48-1.90)$ & 0.910 \\
\hline $\begin{array}{l}\geq 20 \text { cigarettes ever in all } \\
\text { subjects, } n(\%)\end{array}$ & $40(30.1)$ & $17(28.3)$ & $23(31.5)$ & $0.86(0.41-1.81)$ & 0.691 \\
\hline Phototype number & $2.4 \pm 0.7$ & $2.5 \pm 0.7$ & $2.3 \pm 0.6$ & - & $0.146^{\mathrm{a}}$ \\
\hline Phototype 1 and 2, n (\%) & 77 (57.9) & $30(50.0)$ & $47(64.4)$ & $0.55(0.28-1.11)$ & $0.095^{\mathrm{g}}$ \\
\hline Nevi $>50, \mathrm{n}(\%)$ & $66(49.6)$ & $35(58.3)$ & $31(42.5)$ & $1.90(0.95-3.79)$ & $0.069^{\mathrm{g}}$ \\
\hline Burns over $5, \mathrm{n}(\%)$ & $71(53.4)$ & $32(53.3)$ & $39(53.4)$ & $1.00(0.50-1.98)$ & 0.992 \\
\hline NMetM, n (\%) & $72(54.1)$ & $34(56.7)$ & $38(52.1)$ & $1.20(0.61-2.39)$ & 0.595 \\
\hline Stage I, n (\%) & $52(39.1)$ & $24(40.0)$ & $28(38.4)$ & $1.07(0.53-2.16)$ & 0.847 \\
\hline Stage II, n (\%) & $19(14.3)$ & $9(15.0)$ & $10(13.7)$ & $1.11(0.42-2.94)$ & 0.831 \\
\hline Stage III, n (\%) & $38(28.6)$ & $19(31.7)$ & $19(26.0)$ & $1.32(0.62-2.80)$ & 0.474 \\
\hline Stage IV, n (\%) & $24(18.0)$ & $8(13.3)$ & $16(21.9)$ & $0.55(0.22-1.39)$ & 0.200 \\
\hline Trunk, n $(\%)$ & $75(56.4)$ & $36(60.0)$ & $39(53.4)$ & $1.31(0.65-2.61)$ & 0.447 \\
\hline Upper limb, n (\%) & $9(6.8)$ & $5(8.3)$ & $4(5.5)$ & $1.57(0.40-6.12)$ & 0.731 \\
\hline Lower limb, n (\%) & $28(21.1)$ & $11(18.3)$ & $17(23.3)$ & $0.74(0.32-1.73)$ & 0.486 \\
\hline Hands/feet, n (\%) & $8(6.0)$ & $3(5.0)$ & $5(6.8)$ & $0.72(0.16-3.13)$ & 0.729 \\
\hline Head/neck, n (\%) & $13(9.8)$ & $5(8.3)$ & $8(11.0)$ & $0.74(0.23-2.39)$ & 0.612 \\
\hline Superficial spreading, n (\%) & $70(52.6)$ & $31(51.7)$ & $39(53.4)$ & $0.93(0.47-1.85)$ & 0.840 \\
\hline Nodular, n (\%) & $45(33.8)$ & $23(38.3)$ & $22(30.1)$ & $1.44(0.70-2.96)$ & 0.320 \\
\hline Acral lentiginous, n (\%) & $5(3.8)$ & $1(1.7)$ & $4(5.5)$ & $0.29(0.03-2.69)$ & 0.378 \\
\hline Lentigo maligna, n (\%) & $2(1.5)$ & $0(-) b$ & $2(2.7)$ & $0.97(0.94-1.01)$ & 0.501 \\
\hline Spitzoide, n (\%) & $5(3.8)$ & $4(6.7)$ & $1(1.4)$ & $5.14(0.56-47.3)$ & 0.174 \\
\hline Others, $\mathrm{n}(\%)$ & $9(6.8)$ & $3(5.0)$ & $6(8.2)$ & $0.59(0.14-2.46)$ & 0.512 \\
\hline Breslow thickness, $\mathrm{mm}$, mean $\pm \mathrm{SD}$ & $2.05 \pm 1.85$ & $2.17 \pm 2.12$ & $1.95 \pm 1.61$ & - & $0.871^{\mathrm{a}}$ \\
\hline Clark I, n $(\%)$ & $2(1.5)^{\mathrm{c}}$ & $0(-)^{b, d}$ & $2(2.8)$ & $0.97(0.93-1.01)$ & 0.501 \\
\hline Clark II, n (\%) & $32(24.4)^{\mathrm{c}}$ & $15(25.4)^{\mathrm{d}}$ & $17(23.6)^{\mathrm{e}}$ & $1.10(0.50-2.45)$ & 0.810 \\
\hline Clark III, n (\%) & $24(18.3)^{\mathrm{c}}$ & $9(15.3)^{\mathrm{d}}$ & $15(20.8)^{\mathrm{e}}$ & $0.68(0.27-1.70)$ & 0.411 \\
\hline Clark IV, n (\%) & $67(51.1)^{\mathrm{c}}$ & $33(55.9)^{\mathrm{d}}$ & $34(47.2)^{\mathrm{e}}$ & $1.42(0.71-2.83)$ & 0.321 \\
\hline Clark V, n (\%) & $4(3.1)^{c}$ & $1(1.7)^{\mathrm{d}}$ & $3(4.2) \mathrm{e}$ & $0.40(0.04-3.92)$ & 0.627 \\
\hline Ulceration, n (\%) & $51(38.3)$ & $26(43.3)$ & $25(34.2)$ & $1.47(0.73-2.97)$ & 0.284 \\
\hline Mitosis > $1, \mathrm{n}(\%)$ & $83(63.4)^{\mathrm{c}}$ & $39(66.1)^{\mathrm{d}}$ & $44(61.1)^{\mathrm{e}}$ & $1.24(0.60-2.54)$ & 0.555 \\
\hline Regression, $\mathrm{n}(\%)$ & $20(15.3)^{\mathrm{c}}$ & $9(15.3)^{\mathrm{d}}$ & $11(15.3)^{\mathrm{e}}$ & $1.00(0.38-2.60)$ & 0.997 \\
\hline Brisk positive TILs, $\mathrm{n}(\%)$ & $38(29.0)^{\mathrm{c}}$ & $17(28.8)^{\mathrm{d}}$ & $21(29.2)^{\mathrm{e}}$ & $0.98(0.46-2.10)$ & 0.965 \\
\hline Non-brisk TILs, n (\%) & $47(35.9)^{\mathrm{c}}$ & $25(42.4)^{\mathrm{d}}$ & $22(30.6)^{\mathrm{e}}$ & $1.67(0.81-3.43)$ & 0.161 \\
\hline
\end{tabular}


Table III. Continued.

\begin{tabular}{lccccc}
\hline Characteristics & $\begin{array}{c}\text { All patients with } \\
\text { melanoma }(\mathrm{n}=133)\end{array}$ & $\begin{array}{c}\text { 2/L genotype } \\
(\mathrm{n}=60)\end{array}$ & $\begin{array}{c}\text { Non-2/L } \\
\text { genotypes }(\mathrm{n}=73)\end{array}$ & $\begin{array}{c}\text { OR }(\mathrm{CI}), \\
\text { 2/L vs. Non-2/L }\end{array}$ & $\begin{array}{c}\text { P-value, } \\
\text { 2/L vs. Non-2/L }\end{array}$ \\
\hline TILs absence, $\mathrm{n}(\%)$ & $45(34.4)^{\mathrm{c}}$ & $17(28.8)^{\mathrm{d}}$ & $28(38.9)^{\mathrm{e}}$ & $0.64(0.30-1.33)$ & 0.227 \\
Microsatellitosis, $\mathrm{n}(\%)$ & $5(3.8)^{\mathrm{c}}$ & $2(3.4)^{\mathrm{d}}$ & $3(4.2)^{\mathrm{e}}$ & $0.81(0.13-5.00)$ & 1.000 \\
Epithelioid variant, $\mathrm{n}(\%)$ & $37(28.0)^{\mathrm{f}}$ & $14(23.3)$ & $23(31.9)^{\mathrm{e}}$ & $0.65(0.30-1.41)$ & 0.273 \\
Fusate variant, $\mathrm{n}(\%)$ & $13(9.8)^{\mathrm{f}}$ & $6(10.0)$ & $7(9.7)^{\mathrm{e}}$ & $1.03(0.33-3.25)$ & 0.957 \\
Small cell variant, $\mathrm{n}(\%)$ & $2(1.5)^{\mathrm{f}}$ & $0(-)^{\mathrm{b}}$ & $2(2.8)^{\mathrm{e}}$ & $0.97(0.93-1.01)$ & 0.500 \\
More than 1 melanoma, $\mathrm{n}(\%)$ & $20(15.0)$ & $10(16.7)$ & $10(13.7)$ & $1.26(0.49-3.26)$ & 0.634 \\
Additional non-melanoma skin & $22(16.5)$ & $7(11.7)$ & $15(20.5)$ & $0.51(0.19-1.35)$ & 0.170 \\
cancer, n (\%) & & & & & \\
Additional non-skin cancer, $\mathrm{n}(\%)$ & $29(21.8)$ & $11(18.3)$ & $18(24.7)$ & $0.69(0.29-1.59)$ & 0.379 \\
Concurrent thyroid disease, $\mathrm{n}(\%)$ & $16(12.0)$ & $8(13.3)$ & $8(11.0)$ & $1.25(0.44-3.56)$ & 0.675 \\
Melanoma familiarity, $\mathrm{n}(\%)$ & $18(13.5)$ & $10(16.7)$ & $8(11.0)$ & $1.62(0.60-4.42)$ & 0.338 \\
\hline
\end{tabular}

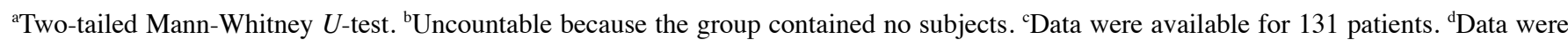
available for 59 patients. ${ }^{e}$ Data were available for 72 patients. fData were available for 132 patients. ${ }^{\text {g}}$ Significant tendencies. BMI, body mass index; FVG, Friuli Venezia-Giulia; IL-1RN, interleukin-1 receptor antagonist gene; OR, odds ratio; TILs, tumor-infiltrating lymphocytes; VNTR, variable number tandem repeat.

melanomas $(\mathrm{OR}=1.77, \mathrm{P}=0.042)$ and non-metastatic melanoma $(\mathrm{OR}=1.90, \mathrm{P}=0.013)$ with healthy controls. Heterozygous $2 / \mathrm{L}$ carriers were at increased risk when comparing NMetM vs. healthy controls $(\mathrm{OR}=2.03, \mathrm{P}=0.006)$, whereas a tendency was observed comparing MetM vs. healthy controls $(\mathrm{OR}=1.68$, $\mathrm{P}=0.063$ ).

Homozygous VNTR $I L-I R N$ 1/1 carriers were at reduced risk comparing melanoma cases with healthy controls $(\mathrm{OR}=0.62, \mathrm{P}=0.017)$. The $1 / 1$ genotype was protective for the subgroup of metastatic melanomas $(\mathrm{OR}=0.58, \mathrm{P}=0.047)$, however, only a tendency for protection was observed for non-metastatic melanomas compared to healthy controls $(\mathrm{OR}=0.65, \mathrm{P}=0.095)$.

By a further analysis, we demonstrated that among melanoma patients the $1 / 2$ and $2 / \mathrm{L}$ genotypes frequencies did not differ between MetM and NMetM patients. The 2/2 genotype was more frequent in MetM (6.6\%) than in NMetM (1.4\%) patients, however, such difference was not statistically significant $(\mathrm{OR}=4.98, \mathrm{P}=0.179)$.

Our study shows in detail that among melanoma patients, the $2 / \mathrm{L}$ genotypes did not differ from the remaining genotypes for demographic characteristics, gender, known risk factors, staging, location, and deepening of melanoma. However, we observed that among melanomas $58.3 \%$ of $2 / \mathrm{L}$ carries had more than 50 body nevi vs. $42.5 \%$ of non-2/L carriers $\mathrm{OR}=1.90, \mathrm{P}=0.069$. This tendency result should be confirmed in enlarged studies. A possible association of $2 / \mathrm{L}$ genotype with an increased number of body nevi could explain the higher risk of $2 / \mathrm{L}$ carriers for melanoma because an elevated body nevi number is a recognized risk factor for melanoma.

This study is the first investigation of VNTR $I L-1 R N$ polymorphism in Italian melanoma patients, and the second one on this polymorphism and melanoma after the study of Broer and colleagues (34). Broer and colleagues performed a study in aggressive melanomas having stage III or higher (in other words all metastatic melanomas) and 343 healthy controls finding a frequency of allele $I L-I R N^{*} 1$ of $73.2 \%$ in melanomas and of $71.7 \%$ in healthy controls, a frequency of allele $I L-1 R N^{*} 2$ of $23.7 \%$ in melanomas and of $28.3 \%$ in healthy controls, whereas the rare allele 3 was found in 2 melanoma patients, and allele 4 was found in 4 melanoma patients. Those data are roughly in line with our allele frequency data. However, the German study (34) did not observe statistically significant differences between aggressive melanomas and healthy controls for VNTR $I L-1 R N$ genotypes; indeed the frequency of $1 / 2$ genotype tended to be even lower in aggressive melanomas than controls $(28.8 \%$ vs. $39.6 \%, \mathrm{P}=0.06)$. At variance, in our Italian melanoma group, the frequency of $1 / 2$ genotype was 1.5 -fold higher (43.6\%) than in German patients. Such a difference could derive by different ethnic background (33) and/or by selection of melanoma cases. Moreover, the high rate of $1 / 2$ genotype in the German healthy controls could be due to different inclusion/exclusion criteria in respect to our study, for example, in our study we excluded high-grade athletes because they have an increased frequency of VNTR $1 / 2$ genotype (37).

The 2/L genotype data of our study are consistent with studies relative to other cancers $(33,47)$. The disproportionate levels of IL-1RA could activate balancing elevation of other factors in the complex network of IL-1 family cytokines and receptors that lead to fine tuning of immune response $(19,48,49)$.

Despite a large number of studies, the association of VNTR $I L-1 R N$ with cancer still shows some inconsistencies (33). The association of the VNTR $I L-1 R N$ polymorphism with cancer was examined by Zhang and colleagues (33) who performed a meta-analysis including 14,854 cases and 19,337 controls from 71 published case-control studies. Genotypic analysis showed significant associations in gastric cancer $(2 / \mathrm{L}$ vs. $\mathrm{L} / \mathrm{L}, \mathrm{OR}=1.22, \mathrm{CI}=1.05-1.41$ ). However, in breast cancer, $2 / \mathrm{L}$ vs. $\mathrm{L} / \mathrm{L}$ was protective $(\mathrm{OR}=0.74, \mathrm{CI}=0.58-0.93)$, whereas in hepatocellular, cervical and lung cancer data were not significant. Moreover, such positive association with cancer 
was stronger in Asian than in Caucasian population (33). The inconsistency is likely caused in part by the differences in the subject ethnicity, sample sizes, disease stages, and cancer types for studies. It is apparent that further studies with large homogeneous patient populations will be needed to validate the association between VNTR $I L-1 R N$ gene polymorphism and human cancer.

Allele 2 of VNTR IL-1RA polymorphism $\left(I L-I R N^{*} 2\right)$ is considered tohavemodulatoryeffects oninflammatory response, however, evidence on final effects associated to $I L-1 R N^{*} 2$ are contradictory $(48,50,51)$. Evaluation of comprehensive effects of $I L-1 R N^{*} 2$ is complicated by the concurrent modulation of the anti-inflammatory IL-1RA and the pro-inflammatory IL-1 cytokine levels $(48,52)$. The presence of the $I L-1 R N^{*} 2$ has been associated with enhanced IL-1 $\beta$ production in vitro (48), and increased inflammatory response $(31,51)$. Indeed, IL-1RA plasma levels are coordinately regulated by both IL-1RA and IL-1beta genes (53) indicating a cross-regulation between the receptor antagonist and IL-1 cytokine expression $(48,49,54)$. Tissue-specific effects are also possible (33).

According to some studies the carriers of $I L-I R N^{*} 2$ have higher IL-1RA levels $(53,55,56)$, and consequently the 2 allele effects could down-regulate IL-1 mediated pro-inflammatory signaling pathways by the IL-1RA blocking of IL-1R1 (37,57,58). A recent study (59) showed that individuals with genotype $2 / 2$ VNTR $I L-1 R N$ exhibited higher IL-1RA expression compared to $1 / 2$ and $1 / 1$ genotypes. The same study indicated that $I L-1 R N^{*} 2$ might be a risk factor for progressive vitiligo (59).

On the basis of such evidence, in $I L-1 R N^{*} 2$ carrier the increased IL-1RA expression could provoke a reduced anti-tumor immune capacity and could favor the onset of melanoma. On the other hand, a study (28) showed that blocking of IL-1R1 by treatment with IL-1R1 neutralizing antibody or IL-1 pathway-specific siRNAs led to growth arrest in IL-1-positive melanoma cells. Furthermore, blocking the IL-1 pathway increased autophagy in IL-1-positive melanoma cells indicating that the endogenous IL-1 system is functional in most human melanoma and interrupting its signaling inhibits the growth of IL-1-positive melanoma cells (28).

It appears plausible that a low IL-1-related immune response (due to moderately elevated IL-1RA as in $2 / \mathrm{L}$ genotypes) increases the risk to develop melanoma, but, at the same time, does not increase or even could reduce the risk to develop an aggressive tumor. Such a hypothesis would fit with our present findings showing that $2 / \mathrm{L}$ genotype increases the risk to develop melanoma, but is not more frequent in metastatic than non-metastatic melanomas. Furthermore, such hypothesis would also fit with Broer et al (34) data showing a tendency of lower frequency of $2 / \mathrm{L}$ in aggressive melanomas than healthy controls.

IL-1R 1 receptor activation by IL- $1 \alpha$ and/or IL- $1 \beta$ induces an array of factors including IL-1, IL-6 (CXCL6), IL-8 (CXCL8), interferon (IFN) $\alpha, \beta$, and $\gamma$, defensins, matrix metalloproteinases (MMPs), C-reactive protein, etc $(18,20,60)$. Thus, the IL-1RA inhibition of IL-1R1 affects several immune factors. It is worth to note that IL-1RA can compete with IL-1 cytokines also for the IL-1R2, which acts as a decoy receptor. Of note, anakinra (a recombinant form of IL-1RA used as an anti-inflammatory drug in certain diseases) or genetic inactivation of the IL-1 $\beta$-IL-1R1 system can lead to less melanoma growth in mice $(18,28)$.

A recent study (61) showed that in MyD88-/- mice and in $\mathrm{C} 57 \mathrm{BL} / 6$ mice treated with anakinra the relapse rates of mice subcutaneous B16 melanoma tumor growth significantly increase. This study suggested that IL-1, via its action on neutrophils, promotes the anti-cancer efficacy of ingenol mebutate (a drug approved for the topical treatment of actinic keratoses that could ultimately also find utility in treating skin cancers), with ingenol mebutate treatment causing both IL- $1 \beta$ induction and IL- $1 \alpha$ release from keratinocytes (61).

Future research should focus on complex gene interactions and biological pathways related to the IL-1 family of cytokines and receptors and melanoma. Improved comprehension of biomolecular immune pathways will support further progress in melanoma management $(1-3,33,36)$.

Study limitations and strengths. A strong point of our study is the highly defined ethnic background of subjects. This variable is important in genetic studies. Specifically, evidence on ethical and geographical variability of genetic polymorphisms in IL-1 family genes is growing $(23,33)$. Moreover, variability in racial distribution and genetic melanoma susceptibility among (and across) different countries suggests that melanoma studies should be performed in restricted and well-characterized ethnic groups $(6,11)$. On the other hand, our results cannot be generalized to populations with different genetic backgrounds. A strength of this study is detailed clinical data of melanoma patients. Limitations of our study include a limited sample size in subgroups of patients and high CIs for some categorical variables. These limitations could have influenced the non-significant results showed in Table II. Thus, future large-scale studies are necessary to better assess the role of such variables.

More personalized approach to cancer is a challenge of current research $(62,63)$. Personalized boosting of anti-tumor immunity in advanced melanomas is a new promising trend in the treatment of malignant melanoma. Immunotherapy is successful for some patients without relapse or progression, but many patients undergoing therapy have progressive disease $(9,64,65)$. Thus, a better understanding of immune factors' modulation contributing to the development of melanoma may increase the likelihood of future improvements in patient management and melanoma prevention by tailored immune modulation modality. IL-1 cytokines and receptors including IL-1RA act in a complex balance, which can both host protect and harm (19). Our data highlighted that in terms of $I L-1 R N$ gene alteration by VNTR in intron 2, IL-1RA homeostasis plays roles in cutaneous melanoma. $I L-1 R N 2 / \mathrm{L}$ (mainly constituted by $1 / 2$ genotype) genotype was found associated to susceptibility to cutaneous melanoma, whereas 1/1 was protective. Thus, our findings support an IL-1 family of receptors contribution to the development of malignant melanoma, suggesting the value of genetic screening as an adjuvant of immune strategies for cancer prevention. Future studies should further explore $I L-I R N$ polymorphisms for their inclusion in risk models for individualized prevention/susceptibility/prognosis in the practice of precision medicine applied in cutaneous melanoma. 
We first suggest that the heterozygous subjects having the short allele $I L-I R N^{*} 2$ are more prone to cutaneous malignant melanoma showing that (innate) immune mechanisms play a role in the susceptibility/pathogenesis of this cancer. Interrelationships of $I L-1 R N$ 86-bp VNTR with other polymorphisms including those of IL-1 $\beta$ gene (IL-1B) are of interest for future research $(40,53) . I L-1 R N$ may be a candidate gene for melanoma pathogenesis or may possibly be a linked marker to other, as yet undefined, genes. However, it is tempting to speculate that determination of IL-1 family polymorphisms could be used in the future for a personalized preventive treatment of healthy subjects at high risk to develop melanoma and/or for prognostic evaluation. Notably, IL-1RA seems to have roles in PD-L1 regulation, which is a main target of immune therapy for advanced melanoma (25). Interestingly, in our study $I L-I R N 2 / \mathrm{L}$ genotype appears to act as a risk factor for melanoma susceptibility independently by conventional risk factors for melanoma, with the possible exception of the elevated presence of nevi. Further investigations are necessary to extend our findings to also examine different ethnic groups (66) and to identify biological pathways related to IL-1RA, which influence skin diseases (67). It is likely that cancer treatment and management will be supplemented in the future by extensive systematic assessment of DNA pathways $(1,35,36)$.

\section{Acknowledgements}

The authors would like to thank Professor Silvio Brusaferro (Department of Medicine, Udine University, Udine, Italy) for his help in enrolment of healthy subjects, and technicians Ms. Patrizia Nacci and Mr. Luca Bazzichetto (Department of Medicine, Udine University, Udine, Italy) for their help with experimental analyses. Lastly, the authors would like to express their gratitude to Professor Carlo Pucillo (Department of Medicine, Udine University, Udine, Italy) for critical reading of the manuscript.

\section{Funding}

The present study was supported by University of Udine grants between 2015 and 2018.

\section{Availability of data and materials}

The datasets used and/or analyzed during the current study are available from the corresponding author on reasonable request

\section{Authors' contributions}

$\mathrm{SC}, \mathrm{CB}, \mathrm{LX}$ and GS were responsible for research creation and design, and provided study material or patients. SC, CB, FR, IS, LX and GS collected, assembled, analysed and interpreted the data, and drafted and finalized the manuscript. All authors read and approved the final version of the manuscript.

\section{Ethics approval and consent to participate}

All experiments were approved by the Ethical Committees of Udine Institutional Ethical Committee (Udine, Italy).

\section{Patient consent for publication}

Not applicable.

\section{Competing interests}

The authors declare that they have no competing interests.

\section{References}

1. Wang LX, Li Y and Chen GZ: Network-based co-expression analysis for exploring the potential diagnostic biomarkers of metastatic melanoma. PLoS One 13: e0190447, 2018.

2. Cauci S, Maione V, Buligan C, Linussio M, Serraino D and Stinco G: BsmI (rs1544410) and FokI (rs2228570) vitamin D receptor polymorphisms, smoking, and body mass index as risk factors of cutaneous malignant melanoma in northeast Italy. Cancer Biol Med 14: 302-318, 2017.

3. La Marra F, Stinco G, Buligan C, Chiriacò G, Serraino D, Di Loreto C and Cauci S: Immunohistochemical evaluation of vitamin D receptor (VDR) expression in cutaneous melanoma tissues and four VDR gene polymorphisms. Cancer Biol Med 14: 162-175, 2017.

4. Gandini S, Montella M, Ayala F, Benedetto L, Rossi CR, Vecchiato A, Corradin MT, DE Giorgi V, Queirolo P, Zannetti G, et al: Sun exposure and melanoma prognostic factors. Oncol Lett 11: 2706-2714, 2016.

5. Bonin S, Albano A, di Meo N, Gatti A, Stinco G, Zanconati F and Trevisan G: Cutaneous melanoma frequencies and seasonal trend in 20 years of observation of a population characterised by excessive sun exposure. Radiol Oncol 49: 379-385, 2015.

6. Ferlay J, Colombet M, Soerjomataram I, Dyba T, Randi G, Bettio M, Gavin A, Visser O and Bray F: Cancer incidence and mortality patterns in Europe: Estimates for 40 countries and 25 major cancers in 2018. Eur J Cancer 103: 356-387, 2018.

7. Newton-Bishop JA, Davies JR, Latheef F, Randerson-Moor J, Chan M, Gascoyne J, Waseem S, Haynes S, O'Donovan C and Bishop DT: 25-Hydroxyvitamin D2/D3 levels and factors associated with systemic inflammation and melanoma survival in the Leeds melanoma cohort. Int J Cancer 136: 2890-2899, 2015.

8. IARC: International Agency for Research on Cancer. http://monographs.iarc.fr/ENG/Classification/. Accessed April 17, 2019.

9. Danielli R, Cisternino F, Giannarelli D, Calabrò L, Camerini R, Savelli V, Bova G, Dragonetti R, Di Giacomo AM, Altomonte M and Maio M: Long-term follow up of metastatic melanoma patients treated with thymosin alpha-1: Investigating immune checkpoints synergy. Expert Opin Biol Ther 18 (Suppl 1): S77-S83, 2018.

10. Isvoranu G, Surcel M, Huică RI, Munteanu AN, Pîrvu IR, Ciotaru D, Constantin C, Bratu O, Neagu M and Ursaciuc C: Natural killer cell monitoring in cutaneous melanoma-new dynamic biomarker. Oncol Lett 17: 4197-4206, 2019.

11. Cecconi L, Busolin A, Barbone F, Serraino D, Chiarugi A, Biggeri A and Catelan D: Spatial analysis of incidence of cutaneous melanoma in the Friuli Venezia Giulia region in the period 1995-2005. Geospat Health 11: 422, 2016.

12. Bray F, Ferlay J, Soerjomataram I, Siegel RL, Torre LA and Jemal A: Global cancer statistics 2018: GLOBOCAN estimates of incidence and mortality worldwide for 36 cancers in 185 countries. CA Cancer J Clin 68: 394-424, 2018.

13. Jiang AJ, Rambhatla PV and Eide MJ: Socioeconomic and lifestyle factors and melanoma: A systematic review. Br J Dermatol 172: 885-915, 2015.

14. EUCAN (2012): Malignant melanoma of the skin. ecoiarcfr/eucan/Canceraspx?Cancer=20. 2012: Accessed April 17, 2019.

15. The Italian Association of Cancer Registries, AIRTUM Working Group. I numeri del cancro in Italia-2018. $2018 \mathrm{http} / / /$ registritumori.it/itacan. Accessed April 17, 2019.

16. AIRTUM (Associazione Italiana Registri Tumori) Working Group. Italian cancer figures-Report 2014. Prevalence and cure of cancer in Italy. Epidemiol Prev 38 (Suppl 1): S1-S144, 2014.

17. Piscitelli P, Neglia C,Falco A, Rivezzi M, Agnello N, Argentiero A, Chitano G, Distante C, Della Rosa G, Vinci G, et al: Melanoma in the Italian population and regional environmental influences: A national retrospective survey on 2001-2008 hospitalization records. Int J Environ Res Public Health 12: 9102-9118, 2015. 
18. Dinarello CA: Overview of the IL 1 family in innate inflammation and acquired immunity. Immunol Rev 281: 8-27, 2018.

19. Khazim K, Azulay EE, Kristal B and Cohen I: Interleukin 1 gene and susceptibility to disease. Immunol Rev 281: 40-56, 2018.

20. Mantovani A, Barajon I and Garlanda C: IL-1 and IL-1 regulatory pathways in cancer progression and therapy. Immunol Rev 281: 57-61, 2018.

21. Malik A and Kanneganti TD: Function and regulation of IL-1 $\alpha$ in inflammatory diseases and cancer. Immunol Rev 281: 124-137, 2018.

22. Garlanda C, Dinarello CA and Mantovani A: The interleukin-1 family: Back to the future. Immunity 39: 1003-1018, 2013.

23. Cauci S, Guaschino S, De Aloysio D, Driussi S, De Santo D, Penacchioni P and Quadrifoglio F: Interrelationships of interleukin-8 with interleukin-1beta and neutrophils in vaginal fluid of healthy and bacterial vaginosis positive women. Mol Hum Reprod 9: 53-58, 2003.

24. Marriott HM, Gascoyne KA, Gowda R, Geary I, Nicklin MJ, Iannelli F, Pozzi G, Mitchell TJ, Whyte MK, Sabroe I and Dockrell DH: Interleukin-1 $\beta$ regulates CXCL8 release and influences disease outcome in response to Streptococcus pneumoniae, defining intercellular cooperation between pulmonary epithelial cells and macrophages. Infect Immun 80: 1140-1149, 2012.

25. Guo B, Zhang $\mathrm{J}$ and Fu S: Inflammasomes/IL-1 pathways in myeloid cells modulate PD-1/PD-L1 checkpoint molecules. J Immunol 198 (Suppl 1): S130.24, 2017.

26. Rotte A, Bhandaru M, Zhou Y and McElwee KJ: Immunotherapy of melanoma: Present options and future promises. Cancer Metastasis Rev 34: 115-128, 2015

27. Coussens LM, Zitvogel L and Palucka AK: Neutralizing tumor-promoting chronic inflammation: A magic bullet? Science 339: 286-291, 2013.

28. Qin Y, Ekmekcioglu S, Liu P, Duncan LM, Lizée G, Poindexter N and Grimm EA: Constitutive aberrant endogenous interleukin-1 facilitates inflammation and growth in human melanoma. Mol Cancer Res 9: 1537-1550, 2011

29. Webb AC, Collins KL, Auron PE, Eddy RL, Nakai H, Byers MG, Haley LL, Henry WM and Shows TB: Interleukin-1 gene (IL1) assigned to long arm of human chromosome 2. Lymphokine Res 5: 77-85, 1986.

30. Nicklin MJ, Weith A and Duff GW: A physical map of the region encompassing the human interleukin-1 alpha, interleukin-1 beta, and interleukin-1 receptor antagonist genes. Genomics 19: 382-384, 1994

31. El-Omar EM, Carrington M, Chow WH, McColl KE, Bream JH, Young HA, Herrera J, Lissowska J, Yuan CC, Rothman N, et al: Interleukin-1 polymorphisms associated with increased risk of gastric cancer. Nature 404: 398-402, 2000.

32. Perri F, Piepoli A, Bonvicini C, Gentile A, Quitadamo M, Di Candia M, Cotugno R, Cattaneo F, Zagari MR, Ricciardiello L, et al: Cytokine gene polymorphisms in gastric cancer patients from two Italian areas at high and low cancer prevalence. Cytokine 30: 293-302, 2005.

33. Zhang Y, Liu C, Peng H, Zhang J and Feng Q: IL1 receptor antagonist gene IL1-RN variable number of tandem repeats polymorphism and cancer risk: A literature review and meta-analysis. PLoS One 7: e46017, 2012.

34. Broer PN, Aung T, Heidekrueger PI, Prantl L and Narayan D: Divisive influence of interleukin-1 receptor antagonist polymorphisms in melanoma patients. Clin Hemorheol Microcirc 67: 319-326, 2017.

35. Sokolenko AP and Imyanitov EN: Molecular tests for the choice of cancer therapy. Curr Pharm Des 23: 4794-4806, 2017.

36. Gu F, Chen TH, Pfeiffer RM, Fargnoli MC, Calista D, Ghiorzo P, Peris K, Puig S, Menin C, De Nicolo A, et al: Combining common genetic variants and non-genetic risk factors to predict risk of cutaneous melanoma. Hum Mol Genet 27: 4145-4156, 2018.

37. Cauci S, Di Santolo M, Ryckman KK, Williams SM and Banfi G: Variable number of tandem repeat polymorphisms of the interleukin-1 receptor antagonist gene IL-1RN: A novel association with the athlete status. BMC Med Genet 11: 29 , 2010.

38. Balch CM, Gershenwald JE, Soong SJ, Thompson JF, Atkins MB Byrd DR, Buzaid AC, Cochran AJ, Coit DG, Ding S, et al: Final version of 2009 AJCC melanoma staging and classification. J Clin Oncol 27: 6199-6206, 2009.

39. Fitzpatrick TB: The validity and practicality of sun-reactive skin types I through VI. Arch Dermatol 124: 869-871, 1988.
40. Cauci S, Di Santolo M, Casabellata G, Ryckman K, Williams SM and Guaschino S: Association of interleukin-1beta and interleukin-1 receptor antagonist polymorphisms with bacterial vaginosis in non-pregnant Italian women. Mol Hum Reprod 13: 243-250, 2007.

41. Colombini A, Brayda-Bruno M, Lombardi G, Croiset SJ, Ceriani C, Buligan C, Barbina M, Banfi G and Cauci S: BsmI, ApaI and TaqI polymorphisms in the vitamin $\mathrm{D}$ receptor gene (VDR) and association with lumbar spine pathologies: An Italian case-control study. PLoS One 11: e0155004, 2016.

42. Zitvogel L, Kepp O, Galluzzi L and Kroemer G: Inflammasomes in carcinogenesis and anticancer immune responses. Nat Immunol 13: 343-351, 2012

43. Dinarello CA: Introduction to the interleukin-1 family of cytokines and receptors: Drivers of innate inflammation and acquired immunity. Immunol Rev 281: 5-7, 2018

44. Tarlow JK, Cork MJ, Clay FE, Schmitt-Egenolf M, Crane AM, Stierle C, Boehncke WH, Eiermann TH, Blakemore AI, Bleehen SS, et al: Association between interleukin-1 receptor antagonist (IL-1ra) gene polymorphism and early and late-onset psoriasis. Br J Dermatol 136: 147-148, 1997.

45. Tarlow JK, Blakemore AI, Lennard A, Solari R, Hughes HN, Steinkasserer A and Duff GW: Polymorphism in human IL-1 receptor antagonist gene intron 2 is caused by variable numbers of an 86-bp tandem repeat. Hum Genet 91: 403-404, 1993.

46. Clay FE, Tarlow JK, Cork MJ, Cox A, Nicklin MJ and Duff GW: Novel interleukin-1 receptor antagonist exon polymorphisms and their use in allele-specific mRNA assessment. Hum Genet 97: 723-726, 1996.

47. Hishida A, Okugawa Y, Morimoto Y, Shirai Y, Okamoto K, Momokita S, Ogawa A, Tanaka K, Nishikawa R, Toiyama Y, et al: Genetic influence of cytokine polymorphisms on the clinical outcome of Japanese gastrointestinal cancer patients in palliative care. Oncol Lett 17: 623-629, 2019.

48. Santtila S, Savinainen K and Hurme M: Presence of the IL-1RA allele $2(\mathrm{IL} 1 \mathrm{RN} * 2)$ is associated with enhanced IL-1beta production in vitro. Scand J Immunol 47: 195-198, 1998.

49. Danis VA, Millington M, Hyland VJ and Grennan D: Cytokine production by normal human monocytes: Inter-subject variation and relationship to an IL-1 receptor antagonist (IL-1Ra) gene polymorphism. Clin Exp Immunol 99: 303-310, 1995.

50. Rafiq S, Stevens K, Hurst AJ, Murray A, Henley W, Weedon MN, Bandinelli S, Corsi AM, Guralnik JM, Ferrucci L, et al: Common genetic variation in the gene encoding interleukin-1-receptor antagonist (IL-1RA) is associated with altered circulating IL-1RA levels. Genes Immun 8: 344-351, 2007.

51. Reiner AP, Wurfel MM, Lange LA, Carlson CS, Nord AS, Carty CL, Rieder MJ, Desmarais C, Jenny NS, Iribarren C, et al: Polymorphisms of the IL1-receptor antagonist gene (IL1RN) are associated with multiple markers of systemic inflammation. Arterioscler Thromb Vasc Biol 28: 1407-1412, 2008.

52. Dewberry R, Holden H, Crossman D and Francis S: Interleukin-1 receptor antagonist expression in human endothelial cells and atherosclerosis. Arterioscler Thromb Vasc Biol 20: 2394-2400, 2000 .

53. Hurme M and Santtila S: IL-1 receptor antagonist (IL-1Ra) plasma levels are co-ordinately regulated by both IL-1Ra and IL-1beta genes. Eur J Immunol 28: 2598-2602, 1998.

54. Buchs N, di Giovine FS, Silvestri T, Vannier E, Duff GW and Miossec P: IL-1B and IL-1Ra gene polymorphisms and disease severity in rheumatoid arthritis: Interaction with their plasma levels. Genes Immun 2: 222-228, 2001.

55. Bioque G, Crusius JB, Koutroubakis I, Bouma G, Kostense PJ, Meuwissen SG and Peña AS: Allelic polymorphism in IL-1 beta and IL-1 receptor antagonist (IL-1Ra) genes in inflammatory bowel disease. Clin Exp Immunol 102: 379-383, 1995.

56. Strandberg L, Lorentzon M, Hellqvist A, Nilsson S, Wallenius V, Ohlsson C and Jansson JO: Interleukin-1 system gene polymorphisms are associated with fat mass in young men. J Clin Endocrinol Metab 91: 2749-2754, 2006.

57. Ramírez-Pérez S, De la Cruz-Mosso U, Hernández-Bello J, Martínez-Bonilla GE, Ramírez-Dueñas MG, Pereira-Suárez AL, Parra Rojas I, Martínez-López E, Macías-Barragán J and Muñoz-Valle JF: High expression of interleukin-1 receptor antagonist in rheumatoid arthritis: Association with IL1RN*2/2 genotype. Autoimmunity 50: 468-475, 2017. 
58. Živković M, Kolić I, Jesić S, Jotić A and Stanković A: The allele 2 of the VNTR polymorphism in the gene that encodes a natural inhibitor of IL-1 $\beta$, IL-1RA is favorably associated with chronic otitis media. Clin Exp Otorhinolaryngol 11: 118-123, 2018.

59. Singh M, Mansuri MS, Jadeja SD, Marfatia YS and Begum R: Association of interleukin 1 receptor antagonist intron 2 variable number of tandem repeats polymorphism with vitiligo susceptibility in Gujarat population. Indian J Dermatol Venereol Leprol 84: 285-291, 2018.

60. Acuner Ozbabacan SE, Gursoy A, Nussinov R and Keskin O: The structural pathway of interleukin 1 (IL-1) initiated signaling reveals mechanisms of oncogenic mutations and SNPs in inflammation and cancer. PLoS Comput Biol 10: e1003470, 2014.

61. Le TT, Skak K, Schroder K, Schroder WA, Boyle GM, Pierce CJ and Suhrbier A: IL-1 contributes to the anti-cancer efficacy of ingenol mebutate. PLoS One 11: e0153975, 2016.

62. Amin MB, Greene FL, Edge SB, Compton CC, Gershenwald JE, Brookland RK, Meyer L, Gress DM, Byrd DR and Winchester DP: The Eighth Edition AJCC Cancer Staging Manual: Continuing to build a bridge from a population-based to a more 'personalized' approach to cancer staging. CA Cancer J Clin 67: 93-99, 2017.

63. Kattan MW, Hess KR, Amin MB, Lu Y, Moons KG, Gershenwald JE, Gimotty PA, Guinney JH, Halabi S, Lazar AJ, et al: American Joint Committee on Cancer acceptance criteria for inclusion of risk models for individualized prognosis in the practice of precision medicine. CA Cancer J Clin 66: 370-374, 2016.
64. Keller HR, Zhang X, Li L, Schaider H and Wells JW: Overcoming resistance to targeted therapy with immunotherapy and combination therapy for metastatic melanoma. Oncotarget 8 : 75675-75686, 2017.

65. Yu Y and Cui J: Present and future of cancer immunotherapy: A tumor microenvironmental perspective. Oncol Lett 16: 4105-4113, 2018.

66. Abbasian MH, Abbasi B, Ansarinejad N, Motevalizadeh Ardekani A, Samizadeh E, Gohari Moghaddam K and Hashemi MR: Association of interleukin-1 gene polymorphism with risk of gastric and colorectal cancers in an Iranian population. Iran J Immunol 15: 321-328, 2018.

67. Qiao J, Jia QN and Jin HZ: Lack of association of the IL-1RN and IL-10 polymorphisms with risk of psoriasis: A meta-analysis. Mol Genet Genomic Med 7: e00512, 2019. Attribution-NonCommercial-NoDerivatives 4.0 International (CC BY-NC-ND 4.0) License. 